\title{
Rapid Domain Wall Motion in Permalloy Nanowires Excited by Spin-Polarized Current Applied Perpendicular to the Nanowire
}

\author{
C. T. Boone, ${ }^{1}$ J. A. Katine,${ }^{2}$ M. Carey,${ }^{2}$ J. R. Childress,${ }^{2}$ X. Cheng, ${ }^{1}$ and I. N. Krivorotov ${ }^{1}$ \\ ${ }^{1}$ Department of Physics and Astronomy, University of California, Irvine, CA 92697 \\ ${ }^{2}$ Hitachi Global Storage Technologies, San Jose, CA 95135
}

(Dated: December 2, 2018)

\begin{abstract}
We study domain wall (DW) dynamics in permalloy nanowires excited by alternating spinpolarized current applied perpendicular to the nanowire. Spin torque ferromagnetic resonance measurements reveal that DW oscillations at a pinning site in the nanowire can be excited with velocities as high as $800 \mathrm{~m} / \mathrm{s}$ at current densities below $10^{7} \mathrm{~A} / \mathrm{cm}^{2}$.

PACS numbers: 75.60.-d, 75.70.-i, 72.25.-b
\end{abstract}

Domains in ferromagnetic nanostructures are candidates for information storage [1, 2] and processing [3]. To be technologically useful, magnetic domains must be easily manipulated. One way this can be accomplished is by moving the magnetic domain wall (DW) separating two oppositely magnetized domains. Since manipulation of individual DWs in nanostructures with magnetic field [4, 5] is technically challenging, DW motion induced by spin torque (ST) from spin-polarized current has emerged as a promising alternative [6]. The effects of ST on DW are most readily studied in the ferromagnetic nanowire geometry with electric current applied along the nanowire [7-15]. However, spin torque exerted on a DW in this geometry is small because the angle between the current polarization direction and magnetization is small everywhere in the DW. As a result, high current density $\left(>10^{8}\right.$ $\left.\mathrm{A} / \mathrm{cm}^{2}\right)$ is required to achieve DW velocities in the technologically useful range of $\sim 10^{2} \mathrm{~m} / \mathrm{s}[16]$.

High DW velocities $(180 \mathrm{~m} / \mathrm{s})$ at relatively small current densities $\left(\leq 5 \times 10^{7} \mathrm{~A} / \mathrm{cm}^{2}\right)$ have been observed in spin valves with current flowing in the plane of the magnetic layers [17]. This high efficiency of the currentinduced DW motion [7] was attributed to transfer of angular momentum from the fixed layer to the DW in the free layer of the spin valve mediated by the component of spin current perpendicular to the layers. Khvalkovskiy et al. [18] also numerically studied DW motion in spin valve nanowires with current flowing perpendicular to the spin valve layers (CPP geometry). This work predicted that DW velocities of $\sim 10^{2} \mathrm{~m} / \mathrm{s}$ can be achieved at current densities of $\sim 10^{7} \mathrm{~A} / \mathrm{cm}^{2}$ in the CPP geometry [19].

In this Letter we make measurements of CPP DW dynamics in the permalloy $\left(\mathrm{Py} \equiv \mathrm{Ni}_{84} \mathrm{Fe}_{16}\right)$ layer of $\mathrm{C}_{50} \mathrm{Fe}_{50} / \mathrm{Cu} / \mathrm{Py}$ spin valves patterned into $5 \mu \mathrm{m}$ long and $90 \mathrm{~nm}$ wide nanowires. The nanowires are defined on metallic $\mathrm{Cu} / \mathrm{Ta} / \mathrm{Ru}$ films serving as bottom leads, and $500 \mathrm{~nm}$ wide $\mathrm{Ta} / \mathrm{Au}$ top leads are used to apply current perpendicular to the spin valve layers as shown in Fig. 1. The device in Fig. 1 is made in a multi-step nanofabrication process starting from a $\mathrm{Ta}(5) / \mathrm{Cu}(30) / \mathrm{Ta}(3) /$ $\mathrm{Cu}(30) / \mathrm{Ta}(5) / \mathrm{Ru}(10) / \mathrm{Cu}(3) / \mathrm{CO}_{50} \mathrm{Fe}_{50}(7) / \mathrm{Cu}(5) /$

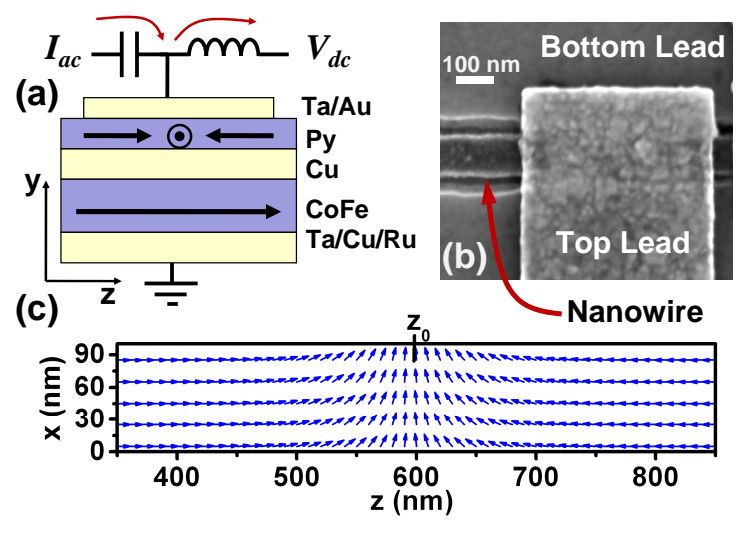

FIG. 1: (a) Schematic of a $\mathrm{Co}_{50} \mathrm{Fe}_{50} / \mathrm{Cu} / \mathrm{Py}$ spin valve in the shape of a $90 \mathrm{~nm}$ wide nanowire with a $500 \mathrm{~nm}$ wide top lead. A domain wall in the Py layer is trapped at a random pinning site under the top lead while the $\mathrm{Co}_{50} \mathrm{Fe}_{50}$ layer is uniformly magnetized. (b) Scanning electron microscopy image of the device, showing the spin valve nanowire, the top lead and the bottom lead. (c) Transverse domain wall profile in the Py nanowire obtained from micromagnetic simulations.

$\mathrm{Py}(3) / \mathrm{Cu}(5) / \mathrm{Ru}(2.5) / \mathrm{Ta}(2.5)$ multilayer (thicknesses in nanometers). The multilayer is deposited onto a thermally oxidized silicon substrate by magnetron sputtering and annealed at $225^{\circ} \mathrm{C}$ for 2 hours. The Py layer magnetization measured with vibrating sample magnetometry $\left(M_{s}=430 \mathrm{emu} / \mathrm{cm}^{3}\right)$ is reduced compared to the bulk value due to magnetically dead layers at the $\mathrm{Cu} / \mathrm{Py}$ interface and interdiffusion of $\mathrm{Cu}$ and $\mathrm{Py}$ [20].

Fig. 2(a) shows the resistance of the device as a function of magnetic field applied parallel to the nanowire. The giant magnetoresistance (GMR) data in Fig. 2(a,b) reveal that the Py layer undergoes reversal in the +200 to -300 Oe field range whereas the $\mathrm{Co}_{50} \mathrm{Fe}_{50}$ layer switches at -900 Oe. Fig. 2(b) shows a major GMR hysteresis loop of the Py layer $( \pm 600 \mathrm{Oe})$. An intermediate resistance (IR) state in this loop arises from DW trapping near the middle of the top lead. The DW is trapped in the same state in a minor hysteresis loop $( \pm 200 \mathrm{Oe})$ in Fig. 2(c). All measurements reported in this Letter are made at $T$ 
$=80 \mathrm{~K}$ in order to prevent thermally-activated depinning of the DW from the IR state observed at $300 \mathrm{~K}$. We expect the DW velocities at $T=300 \mathrm{~K}$ to be similar to those measured at $T=80 \mathrm{~K}[21]$. For the thin-film nanowire geometry of the the Py layer, only a transverse DW is expected to be stable [9]. We confirm this through micromagnetic simulations using OOMMF code [22]. The equilibrium configuration of the DW in the Py layer given by the OOMMF simulations is shown in Fig. 1(c).

We excite motion of the DW trapped in the IR state by applying an alternating current with rms amplitude $I_{a c}$ between the top and the bottom leads of the device. This current applies ac ST to the DW and induces oscillations of the DW position along the nanowire. The DW oscillations give rise to spin valve resistance oscillations, $\delta R_{a c}$, due to the GMR effect, and a rectified voltage, $V_{d c} \sim I_{a c} \delta R_{a c}$, is generated by the device. Measurements of $V_{d c}$ give information on the current-driven DW velocity. In our measurements, we sweep the frequency of the ac current, $f$, in the $0.5-10 \mathrm{GHz}$ range and measure $V_{d c}$ as a function of frequency [23, 24]. The amplitude of the DW oscillations reaches a maximum at the resonance frequency, $f_{0}$, of the DW determined by the strength of the pinning potential, and a resonance peak is observed in the measured response curve $V_{d c}(f)$.

Figure $2(\mathrm{~d})$ shows $V_{d c}(f)$ response curves measured in the middle of the field range where the IR state is stable ( $H=-50$ Oe) with the DW trapped in the pinning potential and at $H=1 \mathrm{kOe}$ with uniform magnetization of both $\mathrm{Py}$ and $\mathrm{Co}_{50} \mathrm{Fe}_{50}$ layers. The -50 Oe response curve shows a resonance at $4 \mathrm{GHz}$ due to the DW oscillations in the pinning potential. We also observe a low-frequency signal, which we attribute to the DW creep in inhomogeneities of the pinning potential [19, 25].

The dependence of the DW velocity on current is given by the measurements of $V_{d c}(f)$ as a function of $I_{a c}$. The maximum DW velocity $v_{\max }=2 \pi f_{0} \Delta z\left(f_{0}\right)$, where $\Delta z\left(f_{0}\right)$ is the amplitude of DW oscillations at resonance, is obtained from the data in Fig. 2(d) [24]:

$$
\Delta z\left(f_{0}\right)=\frac{\sqrt{2} V_{d c}\left(f_{0}\right)}{I_{a c}} \frac{L_{c}}{\Delta R} .
$$

where $L_{c}=500 \mathrm{~nm}$ is the width of the top lead and $\Delta R=37 \mathrm{~m} \Omega$ is the resistance difference between the parallel and antiparallel states of the spin valve.

We compare our data to a theoretical description of DW dynamics in the rigid DW approximation [15]. The motion of magnetization under the influence of ST is described by the Landau-Lifshitz-Gilbert-Slonczewski equation [14, 18, 26]:

$$
\frac{d \vec{m}}{d t}=-\gamma \vec{m} \times\left(\vec{H}_{e}-\frac{\alpha}{\gamma} \frac{d \vec{m}}{d t}+a_{j} \tau(\vec{m} \times \vec{p})+b_{j} \vec{p}\right)
$$

where $\vec{m}$ is the unit vector in the direction of magnetization, $\vec{H}_{e}$ is the effective field, $\alpha$ is the Gilbert damp-
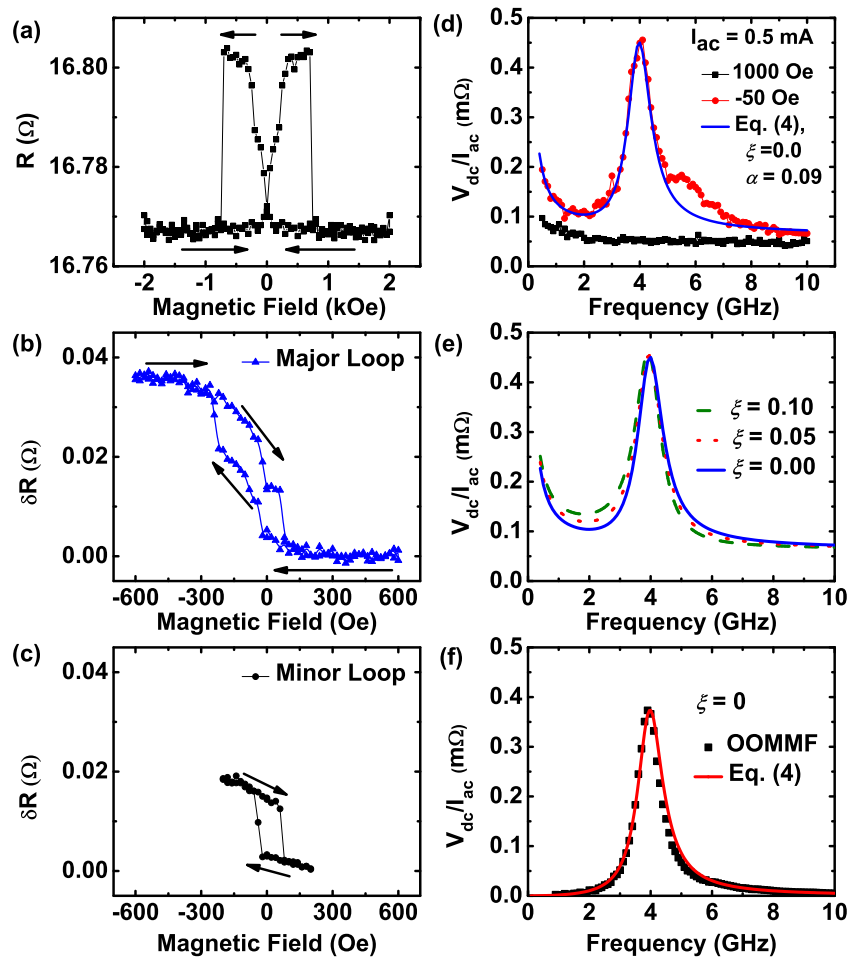

FIG. 2: (a) Magnetoresistance hysteresis loop of the $\mathrm{Co}_{50} \mathrm{Fe}_{50}$ $/ \mathrm{Cu} / \mathrm{Py}$ nanowire spin valve for magnetic field applied parallel to the nanowire axis. The Py layer undergoes magnetization reversal in the +200 to -300 Oe field range whereas the $\mathrm{Co}_{50} \mathrm{Fe}_{50}$ layer switches abruptly at -900 Oe. Major (b) and minor (c) hysteresis loops of the Py layer demonstrate that a DW (intermediate resistance state) can be trapped near the middle of the top lead in a field range from -250 Oe to +100 Oe. (d) $V_{d c}(f)$ response curve measured at -50 Oe with the DW trapped under the top lead and at $1 \mathrm{kOe}$ with uniformly magnetized Py layer. A resonance at $4 \mathrm{GHz}$ is observed when the DW is present under the top lead. The solid line shows a response curve fit calculated from Eq. (4) with an added constant plus $1 / \mathrm{f}$ background. This fit assuming zero field-like torque $(\xi=0)$ gives the best fit parameters $P=0.65, \Lambda=2.0$ and $\alpha=0.09$. (e) Response curves calculated with the same parameters as in (d) except for non-zero values of the fieldlike torque $(\xi>0)$. (f) The response curves calculated from Eq. (44) and from micromagnetic simulations at $I_{a c}=0.5 \mathrm{~mA}$ are nearly identical demonstrating the validity of the rigid DW approximation for small-amplitude DW oscillations.

ing constant of $\mathrm{Py}, a_{j}=\frac{\hbar J P}{2 d e M_{s}}$ is the ST coefficient, $\tau=\frac{2 \Lambda^{2}}{\left(\Lambda^{2}+1\right)+\left(\Lambda^{2}-1\right)(\vec{m} \cdot \vec{p})}[26], \Lambda$ is the ST asymmetry parameter, $J$ is the current density, $P$ is the spin polarization of the current, $d$ is the Py layer thickness, $e$ is the electron charge, $\gamma$ is the gyromagnetic ratio, $\vec{p}$ is the unit vector in the direction of current polarization and $b_{j}=$ $\xi a_{j}$ is the field-like torque coefficient. The dimensionless parameter $\xi$ describes the ratio of the field-like torque to ST [27]. The spatial profile of magnetization in the DW 
is given by [14]:

$$
\begin{array}{r}
m_{z}=\cos (\theta(z))=\tanh \left(\frac{z_{0}-z}{\lambda}\right) \\
m_{x}=\sin (\theta(z)) \cos (\phi)=\operatorname{sech}\left(\frac{z_{0}-z}{\lambda}\right) \cos (\phi)
\end{array}
$$

Here we use a Cartesian system of coordinates with the $z$-axis parallel to the nanowire, the $x$-axis in the plane of the Py film perpendicular to the nanowire, and the $y$-axis along the Py film normal as shown in Fig. 1. In Eq.(3), $z_{0}$ is the coordinate of the DW center, $\lambda$ is the DW width ( $\lambda=53 \mathrm{~nm}$ is given by OOMMF simulations) and $\phi$ is the out-of-plane tilt angle of magnetization at the DW center. In the rigid DW approximation $(\lambda=$ const $)$, Eq. (2) can be rewritten in terms of the DW collective coordinates, $z_{0}$ and $\phi[15,18,28]$ :

$$
\begin{array}{r}
\frac{\dot{z_{0}}}{\lambda}-\alpha \dot{\phi}-\gamma a_{j} \zeta_{1}(\Lambda)=\frac{\gamma}{M_{s}} K \sin (2 \phi) \\
-\alpha \frac{\dot{z_{0}}}{\lambda}-\dot{\phi}+\gamma b_{j}-\gamma a_{j} \zeta_{2}(\Lambda) \tan (\phi)=\gamma \frac{\partial H_{p}}{\partial z} z_{0}
\end{array}
$$

where $K=2 \pi M_{s}^{2}-K_{\perp}$ is the easy-plane shape anisotropy constant, $\frac{\partial H_{p}}{\partial z}$ is the curvature of the pinning potential, $K_{\perp}$ is the easy-axis perpendicular anisotropy constant [29], $\zeta_{1}(\Lambda)=\frac{2 \Lambda^{2} \ln [\Lambda]}{\Lambda^{2}-1}$ and $\zeta_{2}(\Lambda)=\frac{\Lambda(\Lambda-1)}{\Lambda+1}$ describe the effects of ST asymmetry [28]. The value of $K_{\perp} \approx 0.4\left(2 \pi M_{s}^{2}\right)$ is determined from the measurements of resistance versus magnetic field applied perpendicular to the sample plane. Assuming a parabolic pinning potential $\left(\frac{\partial H_{p}}{\partial z}=\right.$ const $)$ and $\phi \ll 1$, Eq. (44) can be solved analytically in the absence of spin torque and damping. This solution describes free oscillations of the DW center, $z_{0}=\Delta z \sin \left(2 \pi f_{0} t\right)$, in the the pinning potential. The resonance frequency of the $\mathrm{DW}, f_{0}$, is determined by the pinning potential curvature [9, 15, 30]:

$$
\frac{\partial H_{p}}{\partial z}=\frac{2 \pi^{2} M_{s} f_{0}^{2}}{\lambda \gamma^{2} K}
$$

The solid line in Fig. 2(d) shows a fit of a theoretical response curve $V_{d c}(f)$ to the experimental data. The theoretical response curve is found by numerically solving Eq. (44) and adding a constant plus $1 / f$ noise background. The fitting parameters used in the fitting procedure are $\alpha, P$ and $\Lambda$. The fit assuming zero field-like torque $(\xi=0)$ gives $P=0.65, \Lambda=2.0$ and $\alpha=0.09$. This value of the damping parameter significantly exceeds the typical Py value $\alpha \approx 0.01$. We directly measure damping of the lowest-energy spin wave mode of the Py nanowire in the state of saturated magnetization using a ST ferromagnetic resonance technique 23, 24, 31 and find $\alpha=0.09$. This shows that the high value of $\alpha$ is an intrinsic property of the ultra-thin Py film and does not originate from emission of spin waves by the moving DW. The observed large $\alpha$ can be explained by diffusion of $\mathrm{Cu}$ into the $\mathrm{Py}$
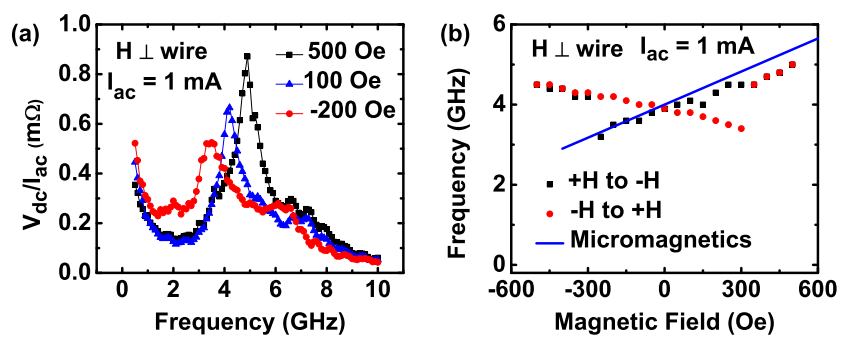

FIG. 3: (a) $V_{d c}(f)$ response curves measured at several magnetic fields, $H_{\perp}$, applied in the plane of the Py layer perpendicular to the nanowire axis. (b) Frequency of the DW resonance as a function of $H_{\perp}$. Solid line is obtained from micromagnetic simulations. The hysteresis in the DW resonance frequency as a function $H_{\perp}$ is due to the field-induced reversal of the DW chirality at $H_{\perp} \approx 350$ Oe.

film known to increase damping [32] and by spin pumping important for ultra-thin ferromagnetic films [33]. The theoretical fit curve in Fig. 2(e) shows deviations from the experimental data at frequencies above the DW resonance. We attribute these deviations to excitation of spin waves in the Py nanowire. We calculate the bottom of the dispersion relation of the lowest-frequency spin wave mode in the Py nanowire to be at $5.5 \mathrm{GHz}$ 31] in agreement with the data in Fig. 2(d).

Figure 2(e) shows theoretical response curves for a nonzero field-like term $(\xi>0)$ in the equations of motion. Our calculations show that a non-zero field-like torque adds an antisymmetric Lorentzian component to the response curve and decreases the quality of the theoretical fit to the experimental data. Fig. 2(e) illustrates that theoretical $V_{d c}(f)$ curves with $\xi>0.1$ show substantial deviations from our experimental data and thus our measurements set an upper limit on the value of field-like torque in our spin valve structure.

We also compare our experimental data to results of micromagnetic simulations. The squares in Fig. 2(f) give the response curve obtained from OOMMF simulations. In these simulations, a spatially-nonuniform field $H_{p}=-\frac{\partial H_{p}}{\partial z} z$ that mimics the $4 \mathrm{GHz}$ pinning potential is applied parallel to the Py nanowire and ST from $I_{a c}$ $=0.5 \mathrm{~mA}$ is applied to drive oscillations of the DW in the nanowire. The solid line in Fig. 2(f) obtained by numerically solving Eq.(4) is in a good agreement with the micromagnetic simulation results.

To further confirm that the observed resonance in $V_{d c}(f)$ is due to DW oscillations and not due to spin wave excitations, we measure the resonance frequency as a function of magnetic field, $H_{\perp}$, applied in the plane of the Py film perpendicular to the nanowire axis. This magnetic field modifies the curvature of the pinning potential, thereby changing the DW resonance frequency [10]. Fig. 3(a) shows $V_{d c}(f)$ measured at several values of $H_{\perp}$, and Fig. 3(b) shows the dependence of $f_{0}$ on 

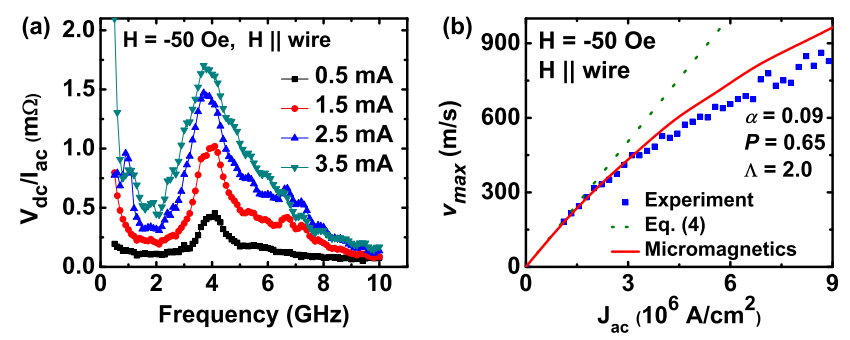

FIG. 4: (a) $V_{d c}(f)$ response curves measured at several values of the excitation current, $I_{a c}$. (b) Maximum speed of the oscillating DW in the pinning potential versus the rms ac drive current density, $J_{a c}$. Squares are the experimental data, dashed line is the rigid DW approximation result given by Eq. (4), solid line is given by micromagnetic simulations.

$H_{\perp}$. The resonance frequency shifts with $H_{\perp}$, and the frequency shift is odd in $H_{\perp}$. This odd frequency shift is a clear signature of the DW resonance for which the sign of the shift is determined by the DW chirality [10]. A frequency shift symmetric with respect to $H_{\perp}$ is expected from spin wave resonances in this geometry and thus the data in Fig.(3) exclude the possibility of spin wave origin of the observed resonance. The hysteretic jumps of $f_{0}$ at $H_{\perp} \approx \pm 350$ Oe correspond to switching of the DW chirality induced by $H_{\perp}$. The dependence of $f_{0}$ on $H_{\perp}$ given by micromagnetic simulations is shown in Fig. 3(b). It is in good agreement with the experimental data.

The dependence of the DW velocity on current is given by measurements of $V_{d c}(f)$ as a function of $I_{a c}$ shown in Fig. 4(a). Fig. 4(b) shows the maximum instantaneous DW velocity, $v_{\max }$, versus current density, $J_{a c}$, calculated from the data in Fig. 4(a) using Eq.(11). We observe velocities as high as $800 \mathrm{~m} / \mathrm{s}$ for $J_{a c}=9 \times 10^{6} \mathrm{~A} / \mathrm{cm}^{2}$. The amplitude of the DW oscillations at $v_{\max }=800 \mathrm{~m} / \mathrm{s}$ is $32 \mathrm{~nm}$ while the smallest DW oscillation amplitude detectable with our technique is $1 \mathrm{~nm}$.

Because the DW mass $\mu \sim M_{s}$ and the ST coefficient $a_{j} \sim 1 / M_{s}$, we expect the DW velocity to scale as $1 / M_{s}^{2}$. The DW velocity at resonance is also expected to be inversely proportional to damping $v_{\max } \sim 1 / \alpha$. Therefore, for standard $\mathrm{Py}\left(\mathrm{Ni}_{80} \mathrm{Fe}_{20}\right)$ material parameters $\left(M_{s}=800 \mathrm{emu} / \mathrm{cm}^{3}, \alpha=0.01\right)$, the expected maximum oscillating DW velocity in the CPP geometry is approximately $2000 \mathrm{~m} / \mathrm{s}$ at the current density of $10^{7}$ $\mathrm{A} / \mathrm{cm}^{2}$. DW velocities as high as $325 \mathrm{~m} / \mathrm{s}$ at a current density of $5 \times 10^{7} \mathrm{~A} / \mathrm{cm}^{2}$ were observed in the currentin-plane geometry for samples with the standard Py material parameters 34 .

The rigid DW approximation predicts a linear increase of the DW velocity with $J_{a c}$ but the experimental data in Fig. 4(b) show clear deviations from the linear behavior for $J_{a c}>1.5 \times 10^{6} \mathrm{~A} / \mathrm{cm}^{2}$. The origin of these deviations is revealed by micromagnetic simulations. The results of these simulations for $v_{\max }\left(J_{a c}\right)$ are shown in Fig. 4(b).
The sublinear dependence of the DW velocity on current similar to that observed in the experiment arises from the breakdown of the rigid DW approximation. The micromagnetic simulations show significant deformations of the DW shape induced by the pinning potential at large amplitudes of DW oscillations.

In conclusion, we make measurements of DW dynamics in Py nanowires excited by spin-polarized current applied perpendicular to the nanowire. In this geometry, we observe DW velocities as high as $800 \mathrm{~m} / \mathrm{s}$ at current densities of $9 \times 10^{6} \mathrm{~A} / \mathrm{cm}^{2}$. The high DW velocities excited by current applied perpendicular to ferromagnetic nanowires are promising for nonvolatile memory and microwave signal processing applications 35]. This work was supported by the NSF Grants DMR-0748810 and ECCS-0701458, and by the Nanoelectronics Research Initiative through the Western Institute of Nanoelectronics.

[1] S. S. P. Parkin et al., Science 320, 190 (2008).

[2] M. Hayashi et al., Science 320, 209 (2008).

[3] D. A. Allwood et al., Science 309, 1688 (2005).

[4] N. L. Schryer and L. R. Walker, J. Appl. Phys. 45, 5406 (1974).

[5] S. Glathe et al., Appl. Phys. Lett. 93, 162505 (2008).

[6] L. Berger, Phys. Rev. B 54, 9353 (1996).

[7] J. Grollier et al., Appl. Phys. Lett. 83, 509 (2003).

[8] A. Yamaguchi et al., Phys. Rev. Lett. 92, 077205 (2004).

[9] G. S. D. Beach et al., J. Magn. Magn. Mater. 320, 1272 (2008).

[10] E. Saitoh et al., Nature 432, 203 (2004).

[11] M. Klaui et al., Phys. Rev. Lett. 94, 106601 (2005).

[12] L. Thomas et al., Nature 443, 197 (2006).

[13] D. Ravelosona et al., Phys. Rev. Lett. 95, 117203 (2005).

[14] A. Thiaville et al., Europhys. Lett. 69, 990 (2005).

[15] G. Tatara et al., Phys. Rep. 468, 213 (2008).

[16] M. Hayashi et al., Phys. Rev. Lett. 98, 037204 (2007).

[17] S. Pizzini et al., Appl. Phys. Express 2, 023003 (2009).

[18] A. V. Khvalkovskiy et al., Phys. Rev. Lett. 102, 067206 (2009).

[19] A. Rebei and O. Mryasov, Phys. Rev. B 74, 014412 (2006).

[20] M. Hecker et al., J. Magn. Magn. Mater. 247, 62 (2002).

[21] E. Martinez et al., Phys. Rev. B 75, 174409 (2007).

[22] M. J. Donahue and D. G. Porter, NIST Interagency Report 6376 (1999).

[23] A. A. Tulapurkar et al., Nature 438, 339 (2005).

[24] J. C. Sankey et al., Phys. Rev. Lett. 96, 227601 (2006).

[25] F. Cayssol et al., Phys. Rev. Lett. 92, 107202 (2004).

[26] J. C. Slonczewski, J. Magn. Magn. Mater. 247, 324 (2002).

[27] A. Brataas et al., Phys. Rep. 427, 157 (2006).

[28] C. T. Boone and I. N. Krivorotov, arXiv:0912.3789 (2009).

[29] J. O. Rantschler et al., J. Appl. Phys. 97, $10 J 113$ (2005).

[30] D. Bedau et al., Phys. Rev. Lett. 101, 256602 (2008).

[31] C. T. Boone et al., Phys. Rev. Lett. 103, 167601 (2009).

[32] Y. Guan et al., J. Appl. Phys. 101, 09D104 (2007).

[33] B. Heinrich et al., Phys. Rev. Lett. 90, 187601 (2003). 
[34] L. Bocklage et al., Phys. Rev. B 78, 180405(R) (2008).

[35] P. M. Braganca et al., IEEE Trans. Nanotech. 8, 190

(2009). 